\section{Clathrin-dependent endocytosis of membrane-bound RANKL in differentiated osteoclasts}

\author{
P. Narducci, R. Bortul, R. Bareggi, \\ V. Nicolin
}

Clinical Department of Biomedicine, University of Trieste, Trieste, Italy

\section{Abstract}

Bone is continuously repaired and remodelled through well-coordinated activity of osteoblasts that form new bone and osteoclasts, which resorb it. Osteoblasts synthesize and secrete two key molecules that are important for osteoclast differentiation, namely the ligand for the receptor of activator of nuclear factor $\kappa B$ (RANKL) and its decoy receptor osteoprotegerin (OPG). Active membrane transport is a typical feature of the resorbing osteoclast during bone resorption. Normally, one resorption cycle takes several hours as observed by monitoring actin ring formation and consequent disappearance in vitro. During these cyclic changes, the cytoskeleton undergoes remarkable dynamic rearrangement. Active cells show a continuous process of exocytosis that plays an essential role in transport of membrane components, soluble molecules and receptor-mediated ligands thus allowing them to communicate with the environment. The processes that govern intracellular transport and trafficking in mature osteoclasts are poorly known. The principal methodological problem that have made these studies difficult is a physiological culture of osteoclasts that permit observing the vesicle apparatus in conditions similar to the in vivo conditions. In the present study we have used a number of morphological approaches to characterize the composition, formation and the endocytic and biosynthetic pathways that play roles in dynamics of differentiation of mature bone resorbing cells using a tri-dimensional system of physiologic coculture.

\section{Introduction}

The dynamic events of osteoclast maturation are interpreted on the basis of the fusion of precursors of the monocyte/macrophage lineage. Osteoclasts $(\mathrm{OC})$ are giant multinucleated cells and their physiological function is to degrade bone matrix: this boneresorbing activity is essential for controlling bone development and turnover, as well as for the control of calcium homeostasis.

The differentiation of $\mathrm{OC}$ is regulated by osteoblasts that are essential regulators of both bone mineralization and osteoclast function. Signals involved in osteoblast physiology include those originating from endocrine hormones, cell contact with bone matrix and neighbouring cell types, soluble signals from osteoclasts and likely bio-mechanical stress. Stromal/osteoblastic cells express two essential molecules for osteoclastogenesis: macrophage colony stimulating factor (MCS-F) and receptor activator of NFkB ligand (RANKL) that is homologous to other TNFR family ligands /TNF, fasL, TRAIL. ${ }^{1}$ The pathway involved in osteoclast differentiation and activation requires two key elements: receptor activator of nuclear factor B (RANK), found in osteoclasts and their precursors, and RANK ligand (RANKL), produced by osteoblasts and stromal cells in the bone marrow. ${ }^{2}$

In addition, M-CSF is required for survival and proliferation of osteoclast precursors. Binding of RANKL to RANK on monocyte/macrophages cells prompts selective intracellular signals that eventuate in the assumption of the osteoclast phenotype. In order to mature, survive and actively resorb bone as a mature osteoclast, the pre-osteoclast must express the receptor RANK and this receptor must engage its ligand RANKL for proper cell maturation.

The resorption function depends by the organization of actin cytoskeleton and the relationship between the F-actin and the plasma membrane in the formation of the sealing-zone. This structure is thought to anchor the osteoclasts to the mineral substrate, generating a cavity that corresponds to the resorption site. ${ }^{3}$ In vitro osteoclasts are able to adhere to various artificial substrates including glass, plastic or synthetic crystals and to form podosomes mimicking the physiological conditions. ${ }^{4}$ Acidification of the cavity between the cell membrane and the bone surface, and the secretion of proteolytic enzymes into it, occurs through a specialized structure, the ruffled border. ${ }^{5}$ Critical components of this complex system are the podosomes, small, actin-associated adhesion sites ${ }^{6}$ that assemble into clusters to belts and develop the sealing zone, that delimits the membrane domain where the ruffled border is formed. Podosomes are typically formed in cells of the monocytic lineage, such as macrophage ${ }^{7}$ osteoclasts $^{8}$ and immature dendritic cells ${ }^{9}$ they represent a continuum of specialized matrix contacts comparable to the complex machinery formed by focal complexes, focal adhesion and fibrillar adhesions. ${ }^{10}$ The presence of podosome rings have also been reported in endothelial cells but their func-
Correspondence: Vanessa Nicolin, Clinical Department of Biomedicine, University of Trieste, Via Manzoni 16, 34138, Trieste, Italy. Tel: +39.040.5586007. Fax: +39.040.5586016. E-mail:nicolin@units.it

Key words: osteoclast, podosomes, ruffled border, intracellular signal, clathrin, endocytosis, receptor activator of NF-kB ligand (RANKL).

Received for publication: 25 September 2009. Accepted for publication: 7 January 2010.

This work is licensed under a Creative Commons Attribution 3.0 License (by-nc 3.0).

CCopyright P. Narducci et al., 2010 Licensee PAGEPress, Italy

European Journal of Histochemistry 2010; $54: e 6$ doi:10.4081/ejh.2010.e6

tion is currently unknown. ${ }^{11,12}$ Podosome-type adhesions are mainly formed in cells that have to cross tissue boundaries such as monocytes. Podosomes are a prominent part of the actin cytoskeleton in osteoclasts, where they form a continuous belt at the cell periphery. ${ }^{13}$ Recent data suggest that both types of structures (podosomes and sealing zone) are formed independently, since osteoclasts form only the sealing zone but not podosomes on the physiological substrate mineralized bone. ${ }^{14}$ The role of podosomes, as opposed to the sealing zone, in bone homeostasis is now open to debate: localized degradation of the surrounding matrix is seen as adhesive and/or protrusive locations in a variety of normal cells and aggressive cancer cells. ${ }^{15}$ Cleavage of matrix components and cytokines, like transforming growth factor $\beta$ (TGF $\beta$ ), can mediate local activation in signalling cascades and further arrangement by new forming adhesion process. ${ }^{16}$

The bone resorption activity of osteoclasts is regulated at many levels, including endocytosis mediated by clathrin, a plasma membrane protein present on the membrane surface that provide a nucleation site for endocytosis. ${ }^{17,18}$

As a model of osteoclastogenesis we used monocytic/macrophage cell line RAW 264.7 type CRL 2278 in accord with our previous reports $^{19,20}$ that demonstrate that this clone undergoes a quicker osteoclast differentiation with respect to the parental clone RAW 264.7 type TIB-71. ${ }^{19}$ This present study aims to clarify the features and the endocytic clathrin-dependent pathway of osteoclast maturation in vitro by a co-culture system in the absolute absence of cytokine and other types of stimulating factors, simulating in vivo physiological behaviour. 


\section{Materials and Methods}

\section{Cell cultures}

As a model system of osteoclastogenesis, we used the murine monocytic/macrophagic CRL2278 cell line (a subclone of RAW 264.7), purchased from American Type Culture Collection (ATCC, Rockville, MD, USA), cocultured with the murine osteoblast cell line CRL-12257 (ATCC).

CRL-2278 and CRL-12257 were cultured in a 24-well plate at a density of 105 cells $/ \mathrm{mL}$ in RPMI-1640 medium with $2 \mathrm{mM}$ L-glutamine and modified to a final concentration of 4.5$\mathrm{g} / \mathrm{L}$ glucose, $1.5 \mathrm{~g} / \mathrm{L}$ sodium bicarbonate, 10 $\mathrm{mM}$ Hepes, $1 \mathrm{mM}$ sodium piruvate and $10 \%$ fetal bovine serum (FBS). Cells were grown for four days then washed once in PBS and fixed for 30 minutes in a solution of $4 \%$ paraformaldehyde.

\section{Tartrate-resistant acid phosphatase cytochemical analysis}

For cytochemical tartrate-resistant acid phosphatase (TRAP) analysis, cells were stained using a leukocyte acid phosphatase kit, according to the manufacture's instruction (Sigma-Aldrich, St Louis, M0, USA). Briefly, cells were washed once in PBS and fixed for 30 min. in a solution of $4 \%$ paraformaldehyde. Fixed cells were then washed in PBS and incubated for 1 hour at $37^{\circ} \mathrm{C}$ in TRAP staining solution. After TRAP reaction, slides were rinsed in deionized water and counterstained with haematoxylin solution for 1-2 min. then dried in air and evaluated microscopically by a Zeiss Axiophot microscope supplied by Photometrics Cool Snap camera (Roper Scientific, Duluth, GA).

\section{Immunofluorescence}

The cells cultured on a coverslip were airdried and fixed in 4\% paraformaldehyde in PBS, washed in PBS (pH 7,2) and blocked by incubation with $5 \%$ NGS, 4\% BSA in PBS. Specimens were incubated overnight at $4^{\circ} \mathrm{C}$ with mouse anti-actin monoclonal antibody (Sigma, Aldrich) and with anti-mouse clathrin diluted 1:50 in blocking buffer and after three washes in PBS, they were incubated with an anti-mouse FITC conjugated secondary antibody diluted 1:100 (Sigma, Aldrich) for 1 hour at $37^{\circ} \mathrm{C}$.

Slides were reacted with $0.1 \mathrm{mg} / \mathrm{mL} 4-6$ diamidino-2 phenylindole diluted in $1 \%$ Tween 20 (DAPI, Sigma) to detect cell nuclei. The slides were washed three times in PBS (pH 7.4), dehydrated in a graded series of ethanol and finally mounted with a solution containing 2.3\% 1,4-diazabicyclo [2.2.2]
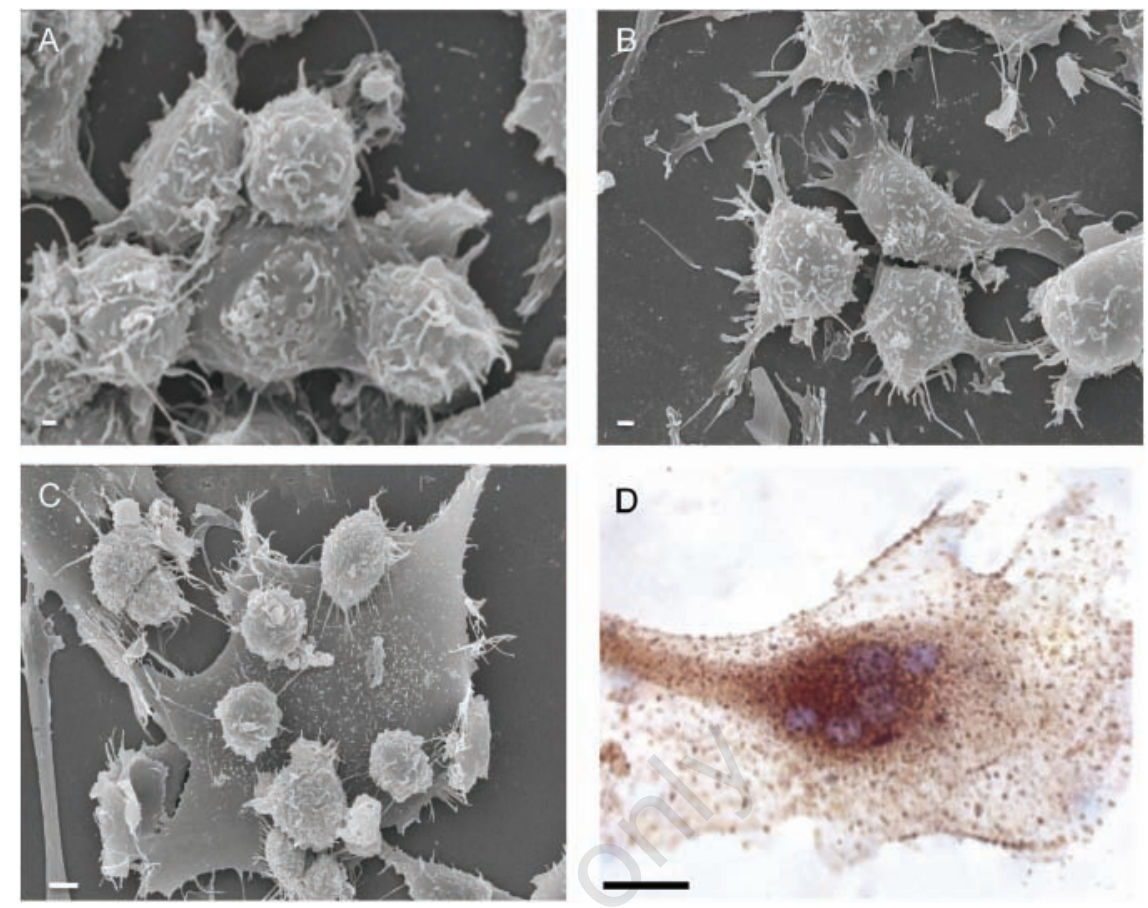

Figure 1. SEM images showing (A) murine monocytes and (B) murine osteoblasts used in the coculture. Osteoclast $(\mathrm{C})$ is formed as the result of cell-cell interactions after 4 days of culture; (D) TRAP immunoenzymatic detection in mature osteoclast. Scale bar: 1A-C: $\mu \mathrm{m}$ 1D: $50 \mu \mathrm{m}$.
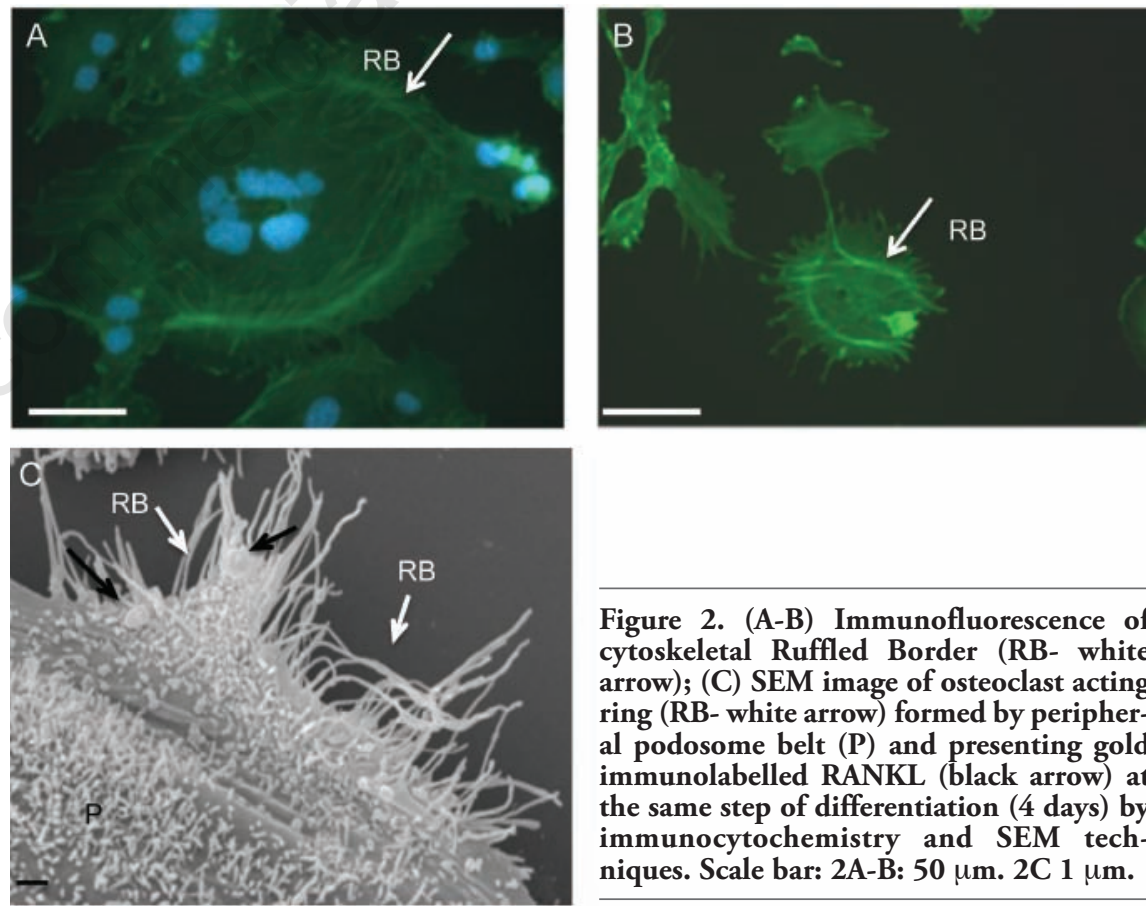

Figure 2. (A-B) Immunofluorescence of cytoskeletal Ruffled Border (RB- white arrow); (C) SEM image of osteoclast acting ring (RB- white arrow) formed by peripheral podosome belt $(\mathrm{P})$ and presenting gold immunolabelled RANKL (black arrow) at the same step of differentiation ( 4 days) by immunocytochemistry and SEM techniques. Scale bar: $2 \mathrm{~A}-\mathrm{B}: 50 \mu \mathrm{m}$. 2C $1 \mu \mathrm{m}$.

octane (DABC0; Sigma) to delay fading. Samples were photographed using a Zeiss Axiophot epifluorescence microscope. Digital images were obtained using a Photometrics Cool SNAP camera (Roper Scientific, Duluth, GA, USA).

\section{Immunoelectron microscopy}

A cell monolayer was sequentially dehydrated in ascending alcohols and infiltrated for 4 hours at room temperature with three changes of LR white acrylic resin hard (Sigma). 
Coverslips were covered with a cylindrical capsule filled with fresh LR white resin and polymerized at $60^{\circ} \mathrm{C}$ for 24 hours. Thin sections were collected on 400-mesh nickel grids, incubated for $30 \mathrm{~min}$. at room temperature in TBS blocking buffer (20 mM Tris-HCl, pH 7.6, 225 $\mathrm{mM} \mathrm{NaCl}, 1 \%$ bovine serum albumin) containing 5\% normal goat serum and then incubated overnight at $4^{\circ} \mathrm{C}$ with a monoclonal antibody against clathrin (clone TD.1, Sigma) heavy chain diluted 1:50 and RANKL (C-20) affinity purified goat polyclonal antibody (Santa Cruz Biotechnology). After $10 \mathrm{~min}$. washing in TBS blocking buffer, samples were incubated for 1 hour at room temperature with EM goat antimouse IgG $10 \mathrm{~nm}$ gold to detect clathrin and EM rabbit anti-goat IgG $15 \mathrm{~nm}$ gold (BBinternational, Cardiff, UK). Control grids were also included in which the primary antibody was omitted. Sections were stained with uranyl acetate.

\section{Analysis of RANKL-expression by immunoblot assay}

Osteoblasts were lysed in lysis buffer (20 mMTris pH 8.0, $2 \mathrm{mM} \mathrm{CaCl}_{2}, 150 \mathrm{mM} \mathrm{NaCl}$, $1 \%$ NP40, 0.1\% SDS) added with protease inhibitors at $4^{\circ} \mathrm{C}$ and centrifuged at $10.000 \mathrm{x} \mathrm{g}$ for $10 \mathrm{~min}$ at $4^{\circ} \mathrm{C}$. The lysates were boiled for 5 min., separated on 7.5\% SDS-PAGE and transferred to polyvinylidene difluoride membranes in transblotting membranes $(20 \mathrm{mM}$ Tris, $150 \mathrm{mM}$ glycine and 20\% methanol pH 8.0 ). The membranes were incubated overnight at $4{ }^{\circ} \mathrm{C}$ with blocking buffer (5\% BSA in $50 \mathrm{mM}$ Tris, $150 \mathrm{mM} \mathrm{NaCl}$ and $10 \mathrm{mM}$ $\mathrm{CaCl}_{2}$ ) and with RANKL anti-mouse IgG (5 $\mu \mathrm{g} / \mathrm{mL}$ ) in TBS containing $5 \%$ non-fat dry milk for lhour. The membrane was then incubated with appropriate secondary antibodies coupled to horseradish peroxidase (1/5.000; Sigma). Then the membranes were washed three times with TBST and TBS, and the signal was visualized with an enhanced chemiluminescence detection system (Amersham, Little Chalfont, UK).

\section{Scanning electron microscopy}

For scanning electron microscopy, cell cultures were fixed with $2.5 \%$ glutaraldehyde in 0.1 buffered phosphate ( $\mathrm{pH} 7.3$ ) for $30 \mathrm{~min}$. at $4^{\circ} \mathrm{C}$, then washed in phosphate buffer and post fixed with $1 \% \mathrm{OsO}_{4}$ in the same buffer for 1 hour at room temperature. The cells were then washed in distilled water, dehydrated in ethanol and dried by the critical-point method with a CPD Balzers Union. Finally, the cultures were sputter-coated with gold and observed under a scanning electron microscope LEIKA Stereoscan 430 i.

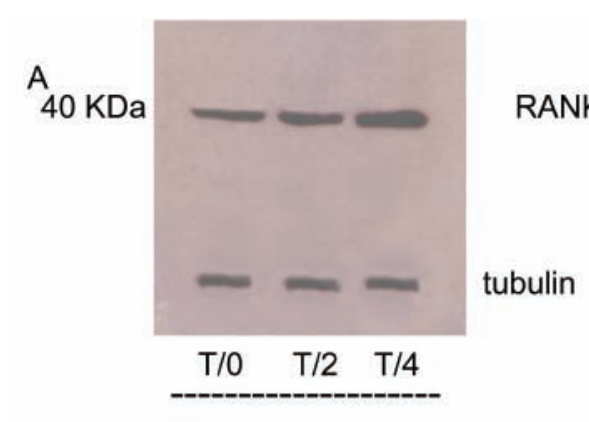

murine osteoblasts
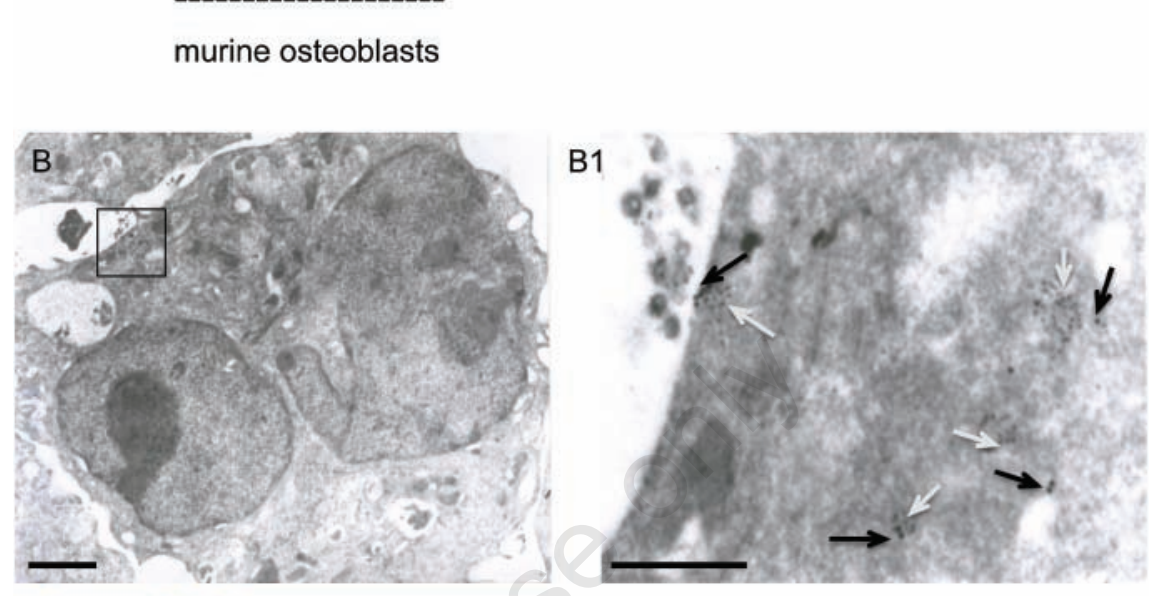

RANKL bulin

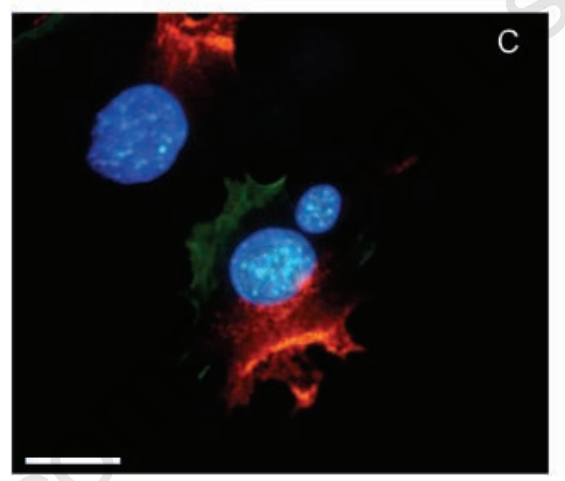

Figure 3. (A) Immunoblot expression of RANK-ligand in cocultured cells. RANK-ligand production results more expressed at $\mathrm{T} / 4$ respect to days $\mathrm{T} / 0-\mathrm{T} / 2$. (3B-B1) Transmission electron microphotographies of immature osteoclast membrane showing the colocalization between RANKI clathrin where RANKl is immunogold labelled with particles of 15 $\mathrm{nm}$ (black arrows) and clathrin clusters are labelled with $10 \mathrm{~nm}$ of gold particles (white arrows). (C) Double labeling clathrin /actin expression on immature osteoclast developed by coculture system. Scale bar: 3A-B1 $1 \mu \mathrm{m}$ 3C: $50 \mu \mathrm{m}$.

\section{Results}

In vitro osteclastogenesis, obtained by murine monocyte/macrophage cell line (RAW 264.7 derived CRL 2278) (Figure 1A) and murine osteoblasts CRL-12257(Figure 1B) (without interaction of specific cytokines), requires about four days to form fully mature bone-resorbing osteoclasts (Figures 1C, 1D, $2 \mathrm{~A})$. The fusion of mononucleated precursors occurs after two to three days. At an early stage of cell culture, osteoclasts exhibit high motility, protruding at the leading edge of moving a broad flat peripheral region of the cell filled with a network of actin filaments (Figure 2B, white arrows). Mature osteoclast cells obtained by the use of our co-culture model resulted as multi-nucleated cells functionally and biologically active as demonstrated positive by tartrate-resistant acid phosphatase assay (TRAP+) (Figure 2A). Moreover they show different surface specialization domains: podosomes, sealing zone and ruffled border. The podosomes are characterized by a dense actin core $(300-400 \mathrm{~nm}$ in diameter and 1-4 $\mu \mathrm{m}$ high) composed of fibres that are oriented perpendicularly to the membrane or connected to the core and then anchored to the membrane. In the sealing zone, that is com- 
posed by a core of filamentous F-actin surrounded by adhesive double ring-like structures and contains the classical adhesion molecules (talin and vinculin), distinct podosomes can be detected as building blocks that form the structure. Such a distribution pattern was demonstrated by fluorescence microscopy by using immunocytochemistry localization as shown in Figure 2A and 2B where we can see the assembling in the actin ring and the formation of the podosome belt respectively. In vitro cultured osteoclasts form the sealing zone-like structure which is about $2 \mu \mathrm{m}$ wide and roughly half as dense, with an average distance between podosomes of 500-140 nm. When the podosomes increase in number and coalesce to form an actin ring in paramarginal cytoplasm, cells may become stationary osteoclasts. Nevertheless the procedure in which podosomes are interconnected remains the same regardless of the substrate; the actin ring rapidly disintegrates during the spreading of osteoclasts and the sealing zone is formed upon transition towards a stationary phase that is coupled with active matrix degradation (Figure 2B and 2C). The ruffled border appears in SEM sections as a finger-like structure that is delimited by membrane folds. These cylindrical extrusions emanate from the membrane with a diameter of 1-2 $\mu \mathrm{m}$ (Figure $2 \mathrm{C}$ ), and almost entirely cover the area that is delimited by the sealing zone and contain actin fibres that support the structure. An accumulation of large and small gold immunoparticles may provide a site of the membrane showing receptorligand complexes (Figure 2C). Physiological RANKL-RANK complexes of our co-culture play an essential role in survival and development of monocyte/macrophage lineage cells versus osteoclast differentiation. The presence of protein RANKL at a weight of $40 \mathrm{kD}$ is demonstrated also by western blotting of murine osteoblast lysate (Figure 3A). Our TEM observations of osteoclast membrane demonstrate that immunogold-labelled RANKL co-localizes with immunogold-labelled clathrin via the clathrin-coated-pit-mediated pathway and both proteins are degraded by lysosome and proteasome pathways (Figure 3B-B1, C). In fact RANKL protein, labelled by immunogold particles of $15 \mathrm{~nm}$ at diameter, is present either on the surface membrane or as internalized complexes. In the same places the clathrin, labelled by immunogold particles of $10 \mathrm{~nm}$ in diameter, co-localizes (Figure 3B-B1). Clathrin-mediated endocytosis showed a significant colocalization, indicating that for RANKL-RANK complexes internalization occurred in a clathrindependent manner. To confirm the TEM data, in Figure 3C, the dual-labelling expression indicated that both Clathrin and RANKL (yellow fluorescence signal due to the overlapping of labelling) had potential association within the superficial zone of the immature osteoclast.

\section{Discussion}

Our previous studies showed that in murine osteoblast and monocyte co-culture, in the absence of exogenous factors such as M-CSF and/or various cytokines, the osteoblasts were able to produce spontaneously RANKL, thus inducing the differentiation of monocyte in morphologically active osteoclasts. ${ }^{20}$ Osteoblasts vary their relative expression of RANKL and OPG to regulate osteoclast formation and function. RANKL is essential to elicit osteoclast development and to inhibit the expression of osteoprotegerin (OPG). Excess RANKL binds to RANK on osteoclasts precursors, allowing the recruitment of tumour necrosis factor receptor-associated factors (TRAFs) at the intracellular domain of RANK. This pathway then regulates osteoclast precursor differentiation into mature osteoclasts and their subsequent activation and survival. Excess OPG binds RANKL and prevents its interaction with RANK thus decreasing numbers and function of osteoclasts. Confocal microscopy has convincingly demonstrated OPG internalization via the clathrin pathway mediated by RANKL in the 293 kidney cell line stably transfected by the full length RANKL mouse protein. ${ }^{18}$ These results translate in our model and underline the importance of the RANKL internalization pathway mediated by clathrin on osteoclast survival and maturation, since the half life modulation on membranous RANKL is then dependent on this binding to the ligand RANK (as well as to OPG when available). Osteoclasts and cells of the osteoblastic lineage can communicate through cell-cell contact to achieve bone remodelling. Although direct contact between mature osteoclasts and mature osteoblasts is controversial, direct contact between mature osteoclasts and bone lining cells have been observed. ${ }^{21}$ Cell-cell contact is associated with polarity of osteoblasts and osteoclasts: the osteoclasts are polarized cells with a ruffled border and a sealing zone at the apical membrane towards extracellular matrix. Usually osteoclasts form podosomes on a variety of substrates and generally need specific signals from bone matrix to induce the formation of sealing zone: of note in osteoclasts derived from our physiological co-culture system the podosome, the sealing zone and the ruffled border are regularly formed. Since clathrin is involved in both cell migration and receptor-mediated endocytosis, it is not surprising that there is presence of a sealing zone and ruffled border with RANKL-RANK clathrin-mediated endocytosis. Interestingly, our results support a clathrin-dependent endocytic pathway that seems to be mainly cytokine-dependent, whereas endocytosis of bone mineral would be a non-clathrin-mediated pathway as supported by previous observations. ${ }^{22}$ Furthermore, OPG can be internalized in several cell types through lipid raft pathways by membranous sydnecan 1 as well as by clathrin-coated formation pathway, depending on the availability of different ligands, as well the concentrations of the receptor. ${ }^{23}$ The clathrin-mediated endocytosis in our model probably reflects either a low syndecan-1 presence on the cell membrane, as well as the canonical behaviour of the receptor-ligand interaction. Our data show that RANKL is detectable both on the cell surface and in the intracellular compartment. One possible explanation for the relatively low amount of RANKL on the cell surface might be that is efficiently endocytosed in a clathrin-dependent manner depending on the availability of ligand in the extra-cellular milieu. These observations suggest a model in which the availability of soluble RANKL derived from the membraneanchored form can be controlled by ectodomain shedding, whereas the levels of RANKL on the cell surface appear to be regulated almost entirely by internalization via clathrincoated pits after contact with cognate molecules. These data are likely to contribute to a better understanding of the local regulation of RANKL and of its role in osteoclastogenesis and the survival and development of monocyte lineage cells implicated in various skeletal and immuno-mediated diseases.

\section{References}

1. Zauli G, Rimondi E, Nicolin V, et al. TNFrelated apoptosis-inducing ligand (TRAIL) blocks osteoclastic differentiation induced by RANKL plus M-CSF. Blood 2004;104: 2044-50.

2. Lacey DL, Timms E, Tan HL, et al. Osteoprotegerin ligand is a cytokine that regulates osteoclast differentiation and activation. Cell 1998;93:165-76.

3. Jurdic P, Saltel F, Chabadel A, Destaing 0 . Podosome and sealing zone: Specificity of the osteoclast model. Eur J Cell Biol 2006; 85:195-2.

4. Akisaka T, Yoshida H, Suzuki R, Takama K. Adhesion structures and their cytoskeleton-membrane interactions at podosomes of osteoclasts in culture. Cell Tissue Res 2008;331:625-41.

5. Mulari M, Vararaniemi J, Vaananen H.K. Intracellular membrane trafficking in bone resorbing osteoclasts. Microsc Res 
Tech2003;61:496-3.

6. Linder S, Aepfelbacher M. Podosomes adhesion hot-spot of invasive cells. Trends Cell Biol 2003;13:376-85.

7. Linder S, Nelson D, Weiss M, Aepfelhofer M. Wiskott-Aldrich syndrome protein regulates podosomes in primary human macrophages. Proc Nat Acad Sci USA 1999; 96:9648-53.

8. Marchisio PC, Cirillo D, Naldini L, et al. Cell-substratum interaction of cultured avian osteoclasts is mediated by specific adhesion structures. J Cell Biol 1984; 99:1696-5.

9. Burns S, Thrasher AJ, Blundell MP, et al. Configuration of human dendritic cells cytoskeleton by Rho GTOases, the WAS protein and differentiation. Blood 2001; 98:1142-9.

10. Linder S, Kopp P. Podosomes at a glance. J Cell Sci 2005;118:2079-82.

11. Moreau V, Tatin F, Varon C, Genot E. Actin can reorganize into podosomes in aortic endothelial cells, a process controlled by Cdc42 and RhoA. Mol Cell Biol 2003; 23:6809-22.
12. Cougoule C, Carreno S, Castander J, et al. Activation of the lysosome-associated p61Hck isoform triggers the biogenesis of podosomes. Traffic 2005; 6:682-94.

13. Luxenburg C, Geblinger D, Klein E, et al. The architecture of the adhesive apparatus of cultured osteoclasts: from podosome formation to sealing zone assembly. PLoS One 2007; 2:e179.

14. Saltel F, Detaing 0, Bard F., Eichert D, Jurdic P. Apatite mediated actin dynamics in resorbing osteoclasts. Mol Biol Vell. 2004; 15:5231-41.

15. Gimona M, Buccione R. Adhesions the mediate invasion. Int J Biochem Cell Biol 2006;38:1875-92.

16. Chang $\mathrm{C}$, Werb $\mathrm{Z}$. The many faces of metalloproteinases: Cell growth, invasion, angiogenesis and metastasis. Trends Cell Biol 2001;11:37-43.

17. Rappaport JZ, Kemal S, Benmerah, Simon SM. Dynamics clathrin and adaptor proteins during endocytosis. Am J Physiol 2006; 291:1072-81.

18. Tat SK, Padrines M, Theoleyre S, et al. OPG/membranous-RANKL complex is internalized via the clathrin pathway before a lysosomal and a proteosomal degradation. Bone 2006;39:706-15.

19. Nicolin V, Ponti C, Narducci P, et al. Different levels of the neuronal nitric oxide synthase isoform modulate the rate of osteoclastic differentiation of TIB-71 and CRL-2278 RAW 264.7 murine cell clones. Anat Rec A Discov Mol Cell Evol Biol 2005; 286:945-54.

20. Nicolin V, Baldini G, Bareggi R, et al. Morphological features of osteoclasts derived from a coculture system. J Mol Histol 2006; 37:171-7.

21. Everts V, Delaisse JM, Korper W, et al. The bone lining cell: its role in cleaning Howship's lacunae and initiating bone formation. J. Bone Miner Res 2002;17:77-90.

22. Pierce A. Lindskog S. Coated pits and vesicles in the osteoclast. J Submicrosc Cytol Pathol 1988; 20:161-7.

23. Tat SK, Pelletier JP, Vlasco CR, et al. New perspective in Osteoarthritis: the OPG and RANKL System as a Potential Therapeutic target. Keio J Med 2009; 58:29-4. 